\title{
Characterization of Interspecific Hybrids in Soybean (Glycine max L.)
}

\author{
Indu Rialch ${ }^{1,2^{*}}$ and Jai Dev Sharma ${ }^{2}$ \\ ${ }^{1}$ Department of Plant Breeding and Genetics, Punjab Agricultural University, \\ Ludhiana-141004, India \\ ${ }^{2}$ Department of Plant Breeding and Genetics, Chaudhary Sarwan Kumar Himachal Pradesh \\ Krishi Vishvavidyalaya, Palampur-176062, India \\ *Corresponding author
}

\begin{tabular}{|l|}
\hline Ke y w or d s \\
Soybean, F1 hybrid, \\
SSR markers \\
\hline Article Info \\
\hline $\begin{array}{l}\text { Accepted: } \\
\text { 20 June } 2019 \\
\text { Available Online: } \\
\text { 10 July } 2019\end{array}$ \\
\hline
\end{tabular}

\section{Introduction}

Soybean (Glycine max [L.] Merr.) is one of the most economically important crops in the world, providing a source of high-quality proteins for feed and food as well as vegetable oil/fuels for human consumption and industrial use (Hartman et al., 2011). It has been widely accepted that soybean was
Successful interspecific hybrids were obtained when we crossed the cultivated soybean with wild annual Glycine soja. Although the success rate was low, whereas, the success rate was zero in case of wild perennial G. tomentella. Probably there is need to improve our crossability skills. Alternately, embryo rescue technique may be employed to recover the hybrids between cultivated soybean and wild perennial species. The hybridity between cultivated and wild annual soybean (Glycine soja) hybrids was confirmed at morphological and molecular level. Molecular markers are useful to confirm the hybridity of interspecific hybrids. SSR markers were used to test $\mathrm{F}_{1}$ hybrid plant obtained from cross of cultivated (Glycine max) and wild species (Glycine soja). Sufficient variability was found for all the traits studied. Seed yield per plant showed significantly positive correlation with reproductive phase, pods per plant, pod length, petiole length, harvest index and 100-seed weight. Significantly negative correlation was observed with plant height and internode length. 
regions for cultivation in agricultural systems. This scenario, i.e., the single origin of cultivated soybeans, appears to be well supported by recent investigation of genomewide diversity among cultivated and wild soybeans at population levels, in which all cultivated accessions were exclusively grouped together into a single clade interior to the G. soja clades (Han et al., 2016; Song et al., 2015; Zhou et al., 2015). Crop relatives have been used for decades for breeding, in particular to transfer genes of resistance or tolerance to pests, diseases or abiotic stress to the cultivated species. Introgression breeding has been extensively used in the genetic improvement of some of the most important Leguminous crops, like soybean.

The genetic base of modern soybean cultivars is narrow since most of the parents used in crossing are from soybean gene pool 1 (GP 1). To guarantee future global food security and sustainable crop production, there exists a need to broaden the genetic base of soybean cultivars. The wild relatives of soybean are a potential reservoir of diversity for this purpose. The perennial Glycine tomentella (Hayata) and the annual Glycine soja (Sieb. and Zucc.) have been hybridized successfully with the domesticated soybean to produce breeding lines suitable for yield testing (Ma and Nelson, 2012; Kabelka et al., 2004; Singh et al., 1990). These wild species are an excellent source of genetic variability, agronomically useful genes, biotic and abiotic stresses. These invaluable traits could be exploited to broaden the genetic base of soybean (Chung and Singh 2008). These species also harbor some undesirable genetic traits, for example, vining, lodging susceptibility, lack of complete leaf abscission, seed shattering and small black coated seeds, however, desirable ones could be sorted out during the course of selection in successive segregating generations. Of the two, the wild progenitor of soybean, G. soja, is the most easily accessible to breeders and has a wealth of diversity preserved in the USDA soybean collection (Carter et al., 2004). Thus, it may be an excellent source of new agronomic genes and traits (Lee et al., 2008). This wild soybean has the same chromosome number as the cultivated soybean, crosses freely via insect or manual hybridization, and progeny are usually completely fertile (Singh and Hymowitz 1988; Weber 1950). Keeping above in view, the present investigation was undertaken, to develop hybrids between Glycine $\max$ and Glycine soja and confirm their hybridity at morphological and molecular level.

\section{Materials and Methods}

The experimental material for present investigation consisted of four cultivated soybean (Glycine max L.) varieties, viz., Bragg, SL-679, PS-1466 and PS-1469 and three wild soybean species (Glycine soja) lines, viz., Glycine soja, Glycine soja (PI 65549) and Glycine soja (PI 366121). The hybridization experiments were conducted at the Experimental Farm, Department of Crop Improvement, CSK HPKV, Palampur during kharif 2011 and 2012 using cultivated genotypes as female and wild species as male. Data were recorded with respect to; total number of soybean flowers pollinated, number of crossed pod set and Per cent pod set. $F_{1}$ hybrids were evaluated during 2012 and 2013. The $F_{1}$ hybrids along with their parents were raised in pots containing mixture of soil, sand and vermi-compost in $2: 1: 1$ ratio in a completely randomized design (CRD) with unequal replications, as number of $F_{1}$ seeds varied for different crosses. For characterization of interspecific crosses involving cultivated and wild species data were recorded for each $F_{1}$ progeny and their parents. The agronomical data obtained from each cross combinations were analysed using the Statistical Analysis System (SAS 
software). Hybridity of $\mathrm{F}_{1}$ plants was confirmed by morphological and molecular markers.

\section{Results and Discussion}

Crossability of cultivated soybean with wild species

Data on number of buds pollinated, pod set and pod set percentage during kharif 2011 and 2012 at Palampur are presented in Table 1. In all, 1282 pollinations were attempted between Glycine $\max$ and Glycine soja. These pollinations resulted in the production of $53 \mathrm{~F}_{1}$ pods. Similary, 2271 pollinations were attempted between Glycine $\max$ and Glycine tomentella.

But the pod set percentage was zero. Difference among the soybean genotypes for crossability has also been reported by Nakayama and Yamaguchi (2002) indicating thereby the presence of genotypic differences for crossability within cultivated soybean.

\section{Confirmation of hybridity of interspecific crosses}

Establishing the true hybrid nature of crosses in the beginning of an experiment is important to develop reliable segregating populations for mapping of genes controlling desirable traits. The $F_{1}$ hybrids showed vigour which was established by their morphological expression.

\section{Confirmation of hybridity at morphological level}

True nature of crosses under study was confirmed in $\mathrm{F}_{1}$ 's for different traits at morphological level as given in Table 2. Leaf and plant morphology of parents and interspecific crosses are shown in Plate 1 (a and b), Plate 2 (a, b, c and d) and Plate 3 (a and $b)$, respectively.

\section{Confirmation of hybridity at molecular level}

The hybridity of four interspecific crosses, viz., Bragg x Glycine soja, SL-679 x Glycine soja (PI-65549), PS-1466 x Glycine soja (PI366121) and PS-1469 x Glycine soja was confirmed at molecular level through four SSR markers.

A total of 34 SSR markers were screened to confirm the hybridity of interspecific crosses. Of these only four markers, viz., Satt301, Salt77, Satt20 and Satt5 were found polymorphic between parents and hybrids and showed robust and reproducible bands as shown in Plate 4 ( $a, b$ and $c)$.

\section{Evaluation of interspecific crosses}

Four interspecific crosses along with their parents were evaluated under field conditions to investigate their actual performance for different agronomic traits. Analysis of variance (Table 3) revealed significant differences among genotypes for all the traits studied, viz., days to $50 \%$ flowering, days to $75 \%$ maturity, reproductive phase, plant height, branches per plant, internode length, nodes per main stem, petiole length, pods per plant, seeds per pod, pod length, biological yield per plant, seed yield per plant, harvest index and 100-seed weight indicating thereby presence of sufficient variability and scope of selection for these traits. Range and mean values of parents and their interspecific crosses for different characters are presented in Table 4.

\section{Simple correlation for yield and related traits}

Yield is a complex character and a function of several component characters. Direct selection based on yield alone will not be very effective in crop improvement programmes. 


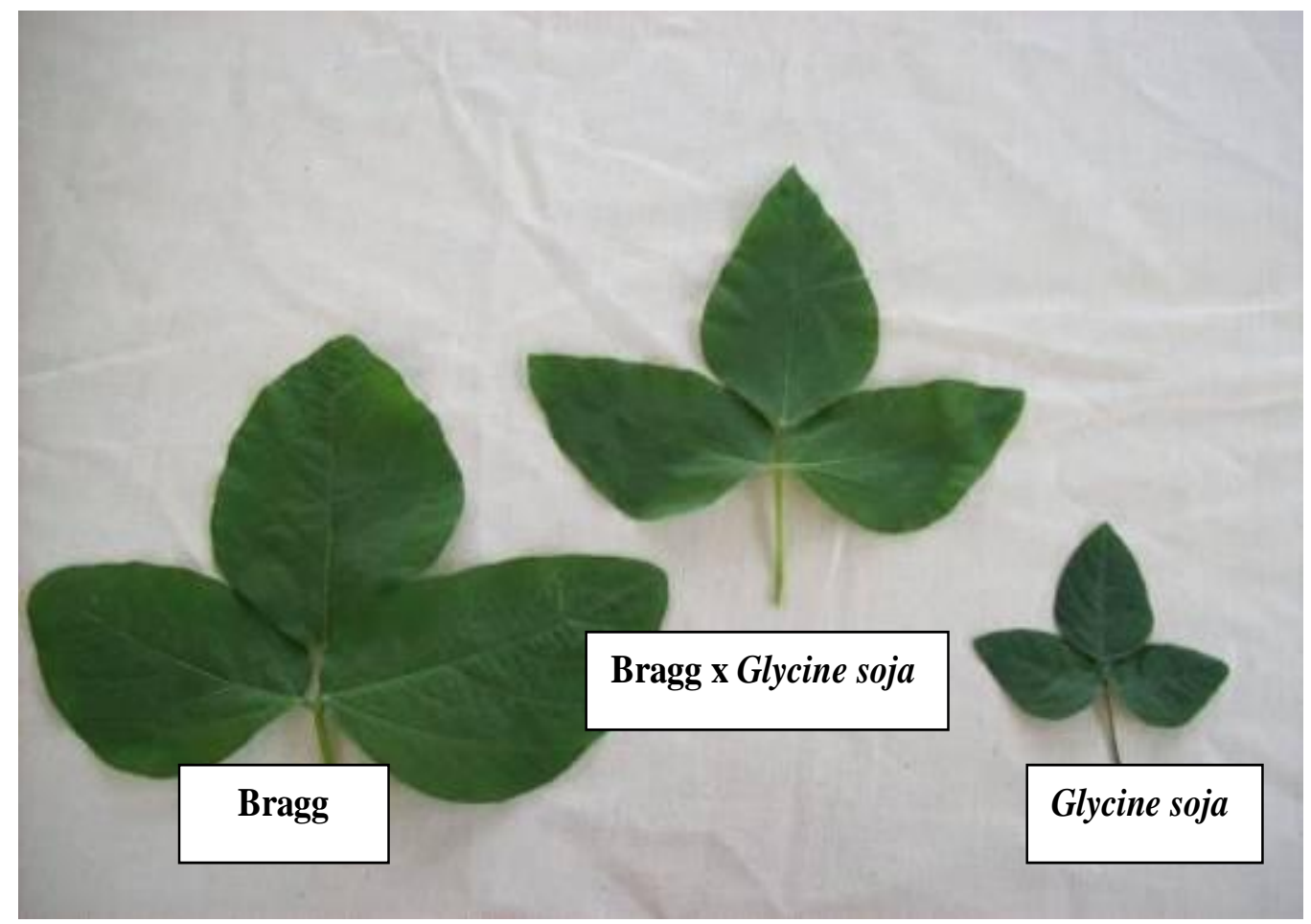

(a)

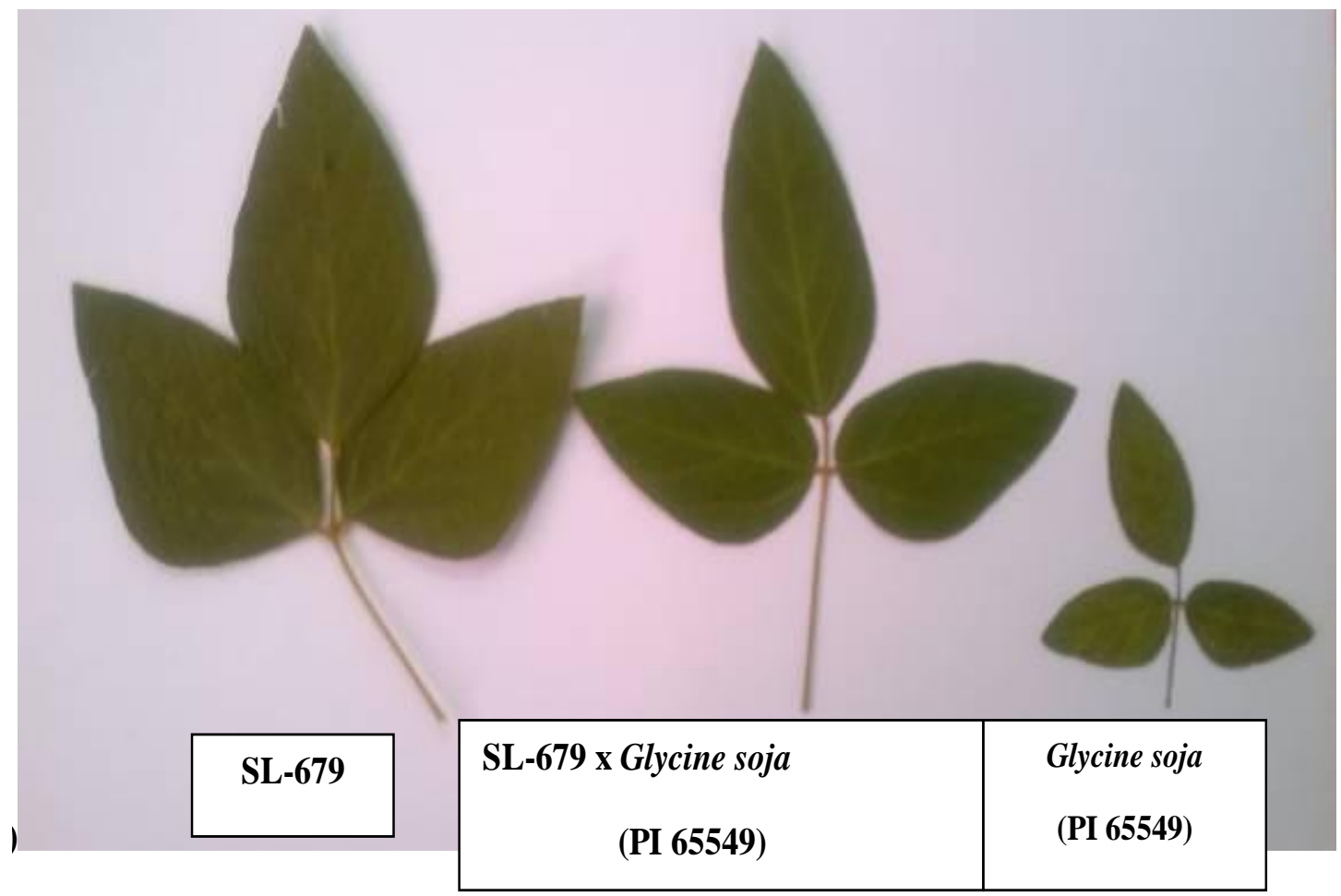

(b)

Plate 1 (a and b) Leaf morphology of parents and interspecific crosses 

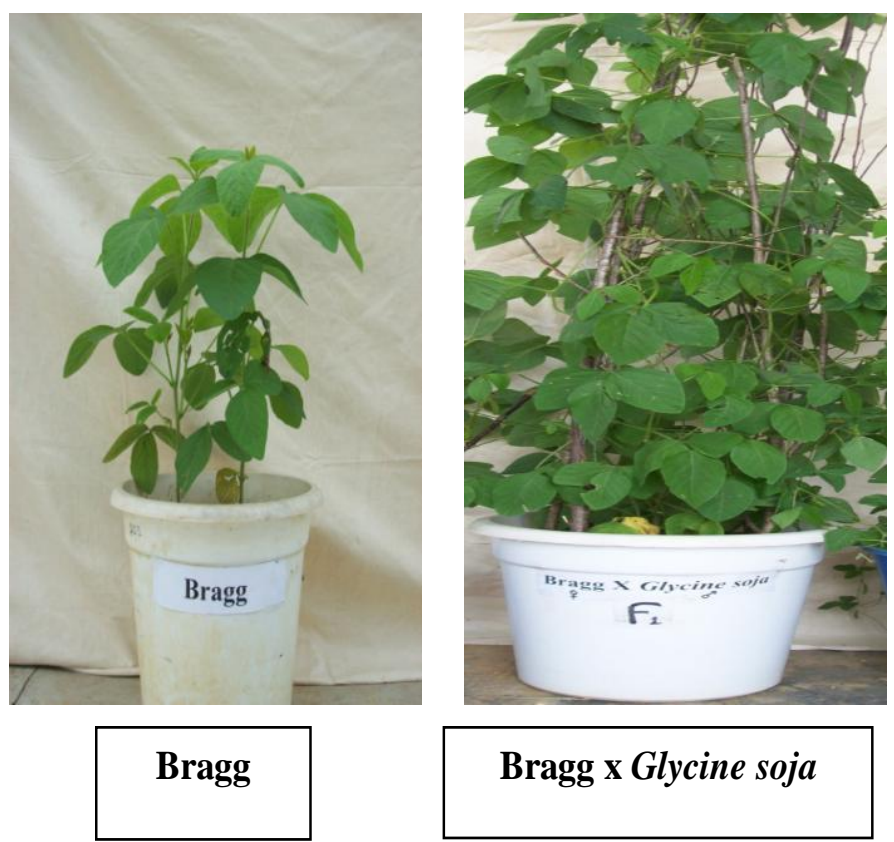

\section{Bragg x Glycine soja}

(a)
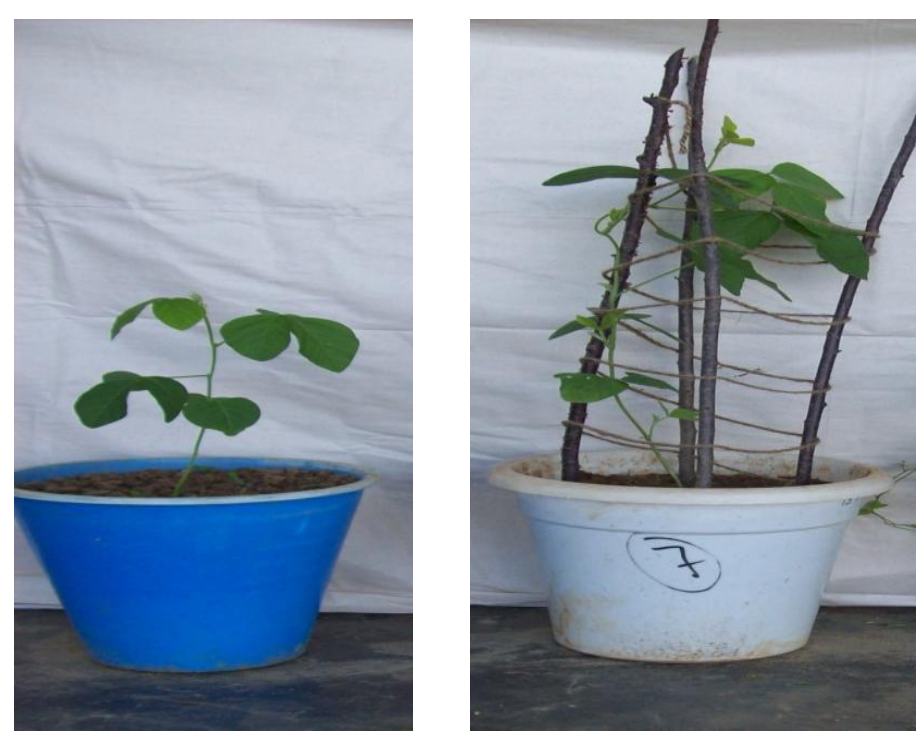

\section{SL-679}

SL-679 x Glycine soja

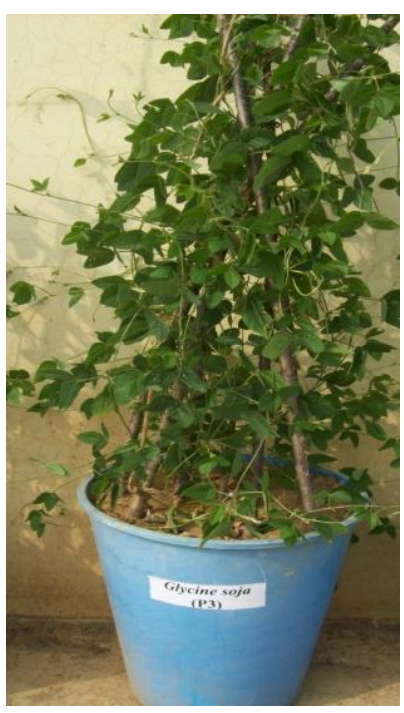

Glycine soja

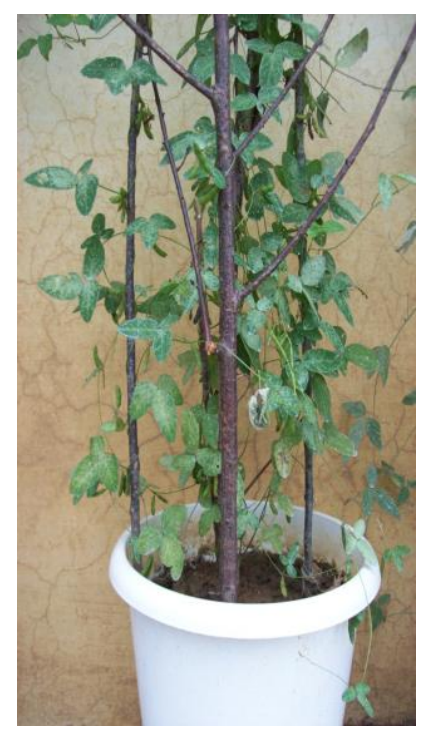

Glycine soja

(b) 

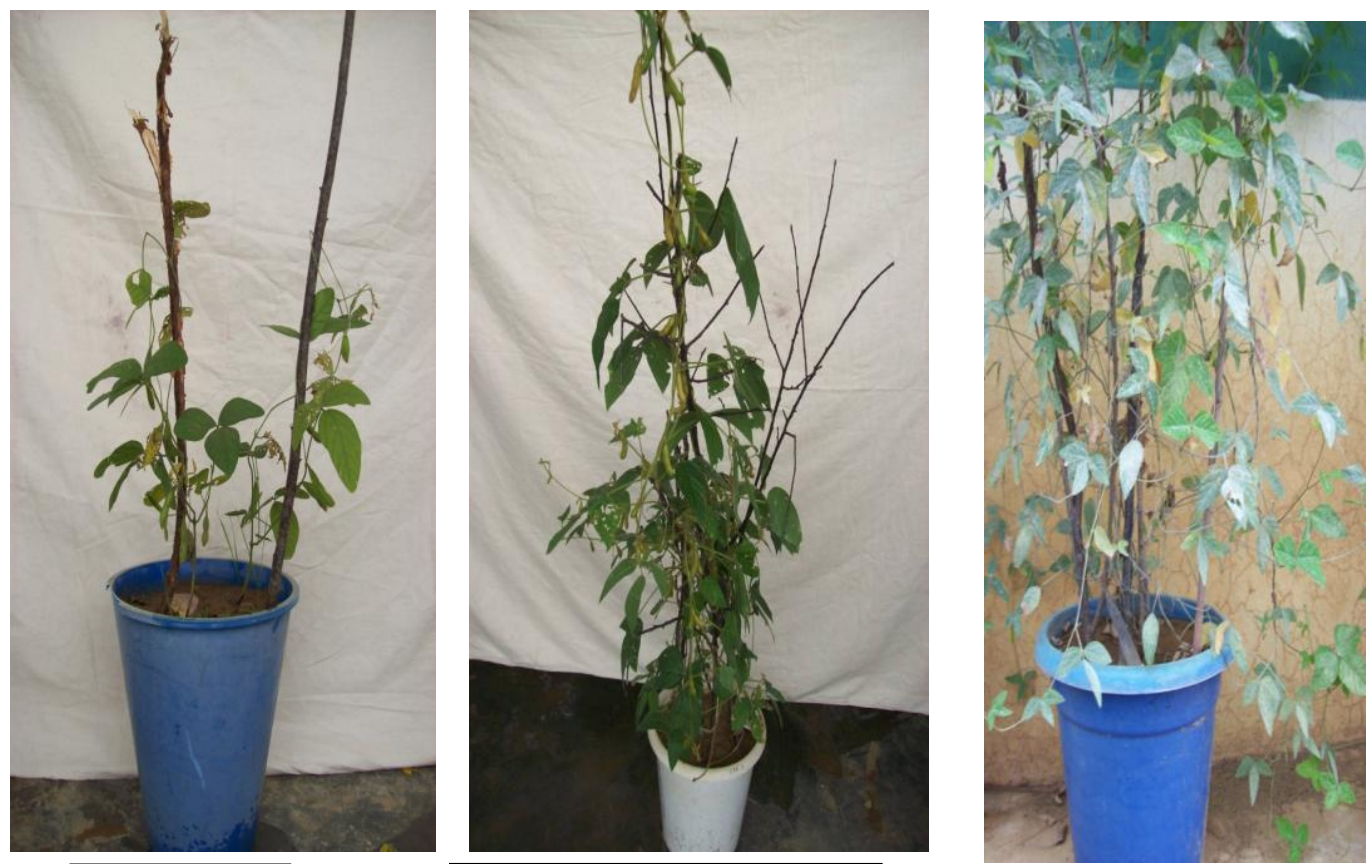

PS-1466

PS-1466 x Glycine soja

G.soja (PI366121)

(c)
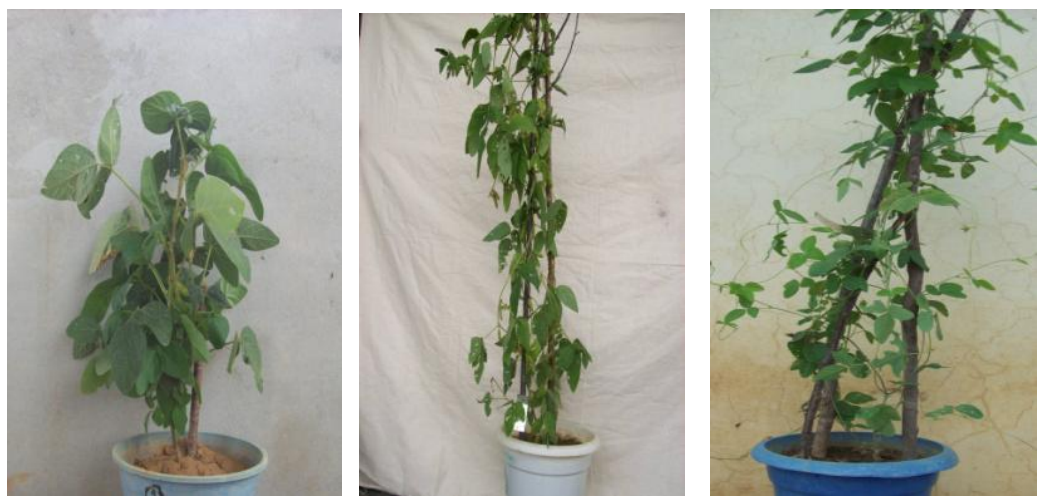

PS-1469

PS-1469 x Glycine soja

Glycine soja

(d)

Plate 2 (a, b, c and d) Plant morphology of parents and interspecific crosses at morphological level 


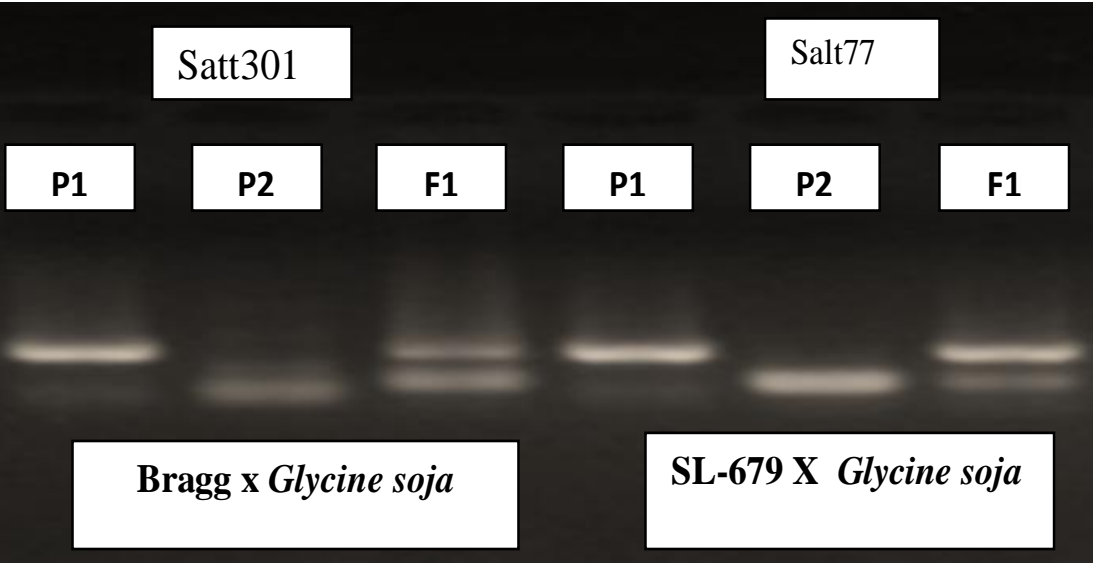

(a)

Satt20

(b)

\section{Satt5}

(c)

PS-1469 x Glycine soja

Plate 3 (a, b and c) Hybridity of parents and interspecific crosses at molecular level 
Table.1 Number of buds pollinated and pod set (\%) during kharif 2011 and 2012 in Glycine max and Glycine soja crosses

\begin{tabular}{|c|c|c|c|c|c|c|}
\hline \multirow[t]{2}{*}{ Cross combinations } & \multicolumn{2}{|c|}{ Number of buds pollinated } & \multicolumn{2}{|c|}{ Number of pod set } & \multicolumn{2}{|c|}{ Pod set $(\%)$} \\
\hline & 2011 & 2012 & 2011 & 2012 & 2011 & 2012 \\
\hline Bragg x G. soja & 298 & 132 & 12 & 7 & 4.02 & 5.30 \\
\hline PS 1466 x G. soja (PI 366121) & 223 & 104 & 9 & 3 & 4.03 & 2.88 \\
\hline SL-679 x G. soja (PI 65549) & 153 & 110 & 8 & 2 & 5.22 & 1.81 \\
\hline PS 1469 x G. soja & 184 & 78 & 8 & 4 & 4.34 & 5.12 \\
\hline Total & 858 & 424 & 37 & 16 & 4.31 & 3.77 \\
\hline
\end{tabular}

Table.2 Morphological characterization of parents and their $\mathrm{F}_{1} \mathrm{~s}$

\begin{tabular}{|c|c|c|c|c|c|c|c|c|c|c|c|c|}
\hline \multirow[t]{3}{*}{ Traits } & \multicolumn{12}{|c|}{ Cross Combinations } \\
\hline & \multicolumn{3}{|c|}{ Bragg X Glycine soja } & \multicolumn{3}{|c|}{ SL-679 X Glycine soja (PI 65549) } & \multicolumn{3}{|c|}{ PS-1469 X Glycine soja } & \multicolumn{3}{|c|}{ PS-1466 X Glycine soja (PI 366121) } \\
\hline & $\mathbf{P}_{1}$ & $\mathbf{P}_{2}$ & $\mathbf{F}_{1}$ & $\mathbf{P}_{1}$ & $\mathbf{P}_{2}$ & $\mathbf{F}_{1}$ & $\mathbf{P}_{1}$ & $\mathbf{P}_{2}$ & $\mathbf{F}_{1}$ & $\mathbf{P}_{1}$ & $\mathbf{P}_{2}$ & $\mathbf{F}_{1}$ \\
\hline $\begin{array}{l}\text { Days to } 50 \% \\
\text { flowering }\end{array}$ & Late & Medium & Late & Late & Medium & Late & Late & Medium & Late & Late & Medium & Late \\
\hline $\begin{array}{l}\text { Plant } \\
\text { Growth type }\end{array}$ & Determinate & Indeterminate & Indeterminate & Determinate & Indeterminate & Indeterminate & Determinate & Indeterminate & Indeterminate & Determinate & Indeterminate & Indeterminate \\
\hline $\begin{array}{l}\text { Plant } \\
\text { Growth } \\
\text { habit }\end{array}$ & Erect & Semi-erect & Semi-erect & Erect & Semi-erect & Semi-erect & Erect & Semi-erect & Semi-erect & Erect & Semi-erect & Semi-erect \\
\hline Plant height & Medium & Tall & Tall & Medium & Tall & Tall & Medium & Tall & Tall & Medium & Tall & Tall \\
\hline Leaf Colour & Green & Dark green & Green & Green & Dark green & Green & Green & Dark green & Green & Green & Dark green & Green \\
\hline $\begin{array}{l}\text { Flower } \\
\text { Colour }\end{array}$ & White & Purple & Purple & White & Purple & Purple & White & Purple & Purple & White & Purple & Purple \\
\hline $\begin{array}{l}\text { Pod } \\
\text { Pubescence }\end{array}$ & Present & Present & Present & Present & Present & Present & Present & Present & Present & Present & Present & Present \\
\hline $\begin{array}{l}\text { Pod } \\
\text { Pubescence } \\
\text { colour }\end{array}$ & $\begin{array}{l}\text { Tawny } \\
\text { (Brown) }\end{array}$ & $\begin{array}{l}\text { Tawny } \\
\text { (Brown) }\end{array}$ & $\begin{array}{l}\text { Tawny } \\
\text { (Brown) }\end{array}$ & $\begin{array}{l}\text { Tawny } \\
\text { (Brown) }\end{array}$ & $\begin{array}{l}\text { Tawny } \\
\text { (Brown) }\end{array}$ & $\begin{array}{l}\text { Tawny } \\
\text { (Brown) }\end{array}$ & $\begin{array}{l}\text { Tawny } \\
\text { (Brown) }\end{array}$ & $\begin{array}{l}\text { Tawny } \\
\text { (Brown) }\end{array}$ & $\begin{array}{l}\text { Tawny } \\
\text { (Brown) }\end{array}$ & $\begin{array}{l}\text { Tawny } \\
\text { (Brown) }\end{array}$ & $\begin{array}{l}\text { Tawny } \\
\text { (Brown) }\end{array}$ & $\begin{array}{l}\text { Tawny } \\
\text { (Brown) }\end{array}$ \\
\hline Pod Colour & Yellow & Black & Brown & Yellow & Black & Brown & Yellow & Black & Brown & Yellow & Black & Brown \\
\hline Seed Shape & Spherical & Elliptical & Spherical & Spherical & Elliptical & Spherical & Spherical & Elliptical & Spherical & Spherical & Elliptical & Spherical \\
\hline Seed Colour & Yellow & Black & Yellow & Yellow & Black & Yellow & Yellow & Black & Yellow & Yellow & Black & Yellow \\
\hline Seed Lustre & Shiny & Dull & Dull & Shiny & Dull & Dull & Shiny & Dull & Dull & Shiny & Dull & Dull \\
\hline $\begin{array}{l}\text { Seed Hilum } \\
\text { colour }\end{array}$ & Black & Grey & Brown & Black & Grey & Brown & Black & Grey & Brown & Black & Grey & Brown \\
\hline $\begin{array}{l}\text { Seed } \\
\text { Cotyledon } \\
\text { colour }\end{array}$ & Yellow & Yellow & Yellow & Yellow & Yellow & Yellow & Yellow & Yellow & Yellow & Yellow & Yellow & Yellow \\
\hline
\end{tabular}


Table.3 Analysis of variance for different traits in soybean

\begin{tabular}{|c|c|c|c|c|}
\hline S. No. & Traits & & [ean sum of s & \\
\hline & & df & Genotypes & Error \\
\hline 1 & Days to $50 \%$ flowering & & $26.39 *$ & 5.25 \\
\hline 2 & Days to $75 \%$ maturity & & $73.63^{*}$ & 9.05 \\
\hline 3 & Reproductive phase & & $0.01 *$ & .001 \\
\hline 4 & Plant height & & $3511.53 *$ & 49.90 \\
\hline 5 & Branches/plant & & $8.23 *$ & 0.92 \\
\hline 6 & Nodes/main stem & & $4.55^{*}$ & 1.53 \\
\hline 7 & Internode length & & $31.60 *$ & 1.24 \\
\hline 8 & Petiole length & & $69.13^{*}$ & 0.43 \\
\hline 9 & Pods/plant & & $1556.29 *$ & 20.74 \\
\hline 10 & Seeds/pod & & $0.06^{*}$ & 0.03 \\
\hline 11 & Pod length & & $0.92 *$ & 0.03 \\
\hline 12 & Biological yield/plant & & $35.95 *$ & 12.12 \\
\hline 13 & Seed yield/plant & & $18.32 *$ & 0.74 \\
\hline 14 & Harvest index & & $170.35^{*}$ & 20.14 \\
\hline 15 & 100-seed wt & & $64.01 *$ & 1.50 \\
\hline
\end{tabular}

* Significant at $\mathrm{P} \leq 0.05$ 
Table.4 Overall range and mean performance of parents and their interspecific crosses for different traits in soybean

\begin{tabular}{|c|c|c|c|c|c|c|c|c|}
\hline Traits & $\mathbf{P}$ & & & 2 & & $\mathbf{F}_{1}$ & & $\mathrm{C}_{1}$ \\
\hline & Range & Mean $\pm \mathbf{S E}$ & Range & Mean \pm SE & Range & Mean $\pm \mathbf{S E}$ & Range & Mean $\pm \mathbf{S E}$ \\
\hline $\begin{array}{l}\text { Days to 50\% } \\
\text { flowering }\end{array}$ & $55.67-64.67$ & $60.58 \pm 0.94$ & $\begin{array}{l}56.67- \\
61.67\end{array}$ & $58.83 \pm 1.81$ & 61.67-64.67 & $62.87 \pm 1.13$ & $63.00-66.00$ & $64.67 \pm 0.88$ \\
\hline $\begin{array}{l}\text { Days to } 75 \% \\
\text { maturity }\end{array}$ & $\begin{array}{l}122.00- \\
129.67\end{array}$ & $124.58 \pm 2.03$ & $\begin{array}{l}115.33- \\
131.67\end{array}$ & $126.42 \pm 1.87$ & $\begin{array}{l}127.00- \\
132.33\end{array}$ & $128.80 \pm 1.48$ & $\begin{array}{l}127.00- \\
132.00\end{array}$ & $129.33 \pm 1.45$ \\
\hline $\begin{array}{l}\text { Reproductive } \\
\text { phase }\end{array}$ & $0.62-0.66$ & $0.64 \pm 0.02$ & $0.45-0.60$ & $0.52 \pm 0.01$ & $0.57-0.59$ & $0.58 \pm 0.009$ & $0.57-0.59$ & $0.58 \pm 0.01$ \\
\hline Plant height & $43.27-54.67$ & $49.12 \pm 5.64$ & $\begin{array}{l}83.33- \\
122.33\end{array}$ & $101.75 \pm 1.76$ & $\begin{array}{l}111.67- \\
132.00\end{array}$ & $123.47 \pm 3.62$ & $\begin{array}{l}120.00- \\
127.00\end{array}$ & $123.33 \pm 2.03$ \\
\hline Branches/plant & $3.93-5.67$ & $5.21 \pm 0.42$ & $6.00-9.67$ & $7.50 \pm 0.68$ & $5.33-7.33$ & $6.53 \pm 0.61$ & $7.00-8.00$ & $7.33 \pm 0.33$ \\
\hline $\begin{array}{l}\text { Nodes/main } \\
\text { stem }\end{array}$ & $8.67-11.13$ & $10.30 \pm 0.58$ & $9.33-13.33$ & $11.00 \pm 0.64$ & $9.67-11.67$ & $10.73 \pm 0.88$ & $11.00-13.00$ & $11.67 \pm 0.67$ \\
\hline $\begin{array}{l}\text { Internode } \\
\text { length }\end{array}$ & $4.15-5.29$ & $4.78 \pm 0.48$ & $7.64-12.02$ & $9.49 \pm 0.67$ & $10.65-13.32$ & $11.68 \pm 0.82$ & $9.23-11.55$ & $10.65 \pm 0.72$ \\
\hline Petiole length & $12.00-12.67$ & $12.33 \pm 0.63$ & $1.83-3.27$ & $2.27 \pm 0.11$ & $2.77-3.27$ & $3.06 \pm 0.13$ & $2.80-3.00$ & $2.90 \pm 0.06$ \\
\hline Pods/plant & $54.53-80.73$ & $67.38 \pm 4.05$ & $17.00-24.33$ & $22.17 \pm 2.98$ & $23.00-25.00$ & $23.67 \pm 1.92$ & $21.00-27.00$ & $23.33 \pm 1.86$ \\
\hline Seeds/pod & $1.91-2.23$ & $2.08 \pm 0.06$ & $1.93-2.37$ & $2.06 \pm 0.16$ & $1.87-2.37$ & $2.06 \pm 0.15$ & $2.00-2.12$ & $2.04 \pm 0.04$ \\
\hline Pod length & $3.50-3.67$ & $3.56 \pm 0.06$ & $2.13-3.00$ & $2.46 \pm 0.12$ & $2.63-3.47$ & $3.13 \pm 0.11$ & $3.00-3.60$ & $3.37 \pm 0.19$ \\
\hline $\begin{array}{l}\text { Biological } \\
\text { yield/plant }\end{array}$ & $28.80-32.93$ & $30.38 \pm 2.33$ & $25.00-31.00$ & $27.08 \pm 2.18$ & $31.00-36.33$ & $33.13 \pm 1.46$ & $35.00-38.00$ & $36.33 \pm 0.88$ \\
\hline $\begin{array}{l}\text { Seed } \\
\text { yield/plant }\end{array}$ & $10.00-15.87$ & $12.68 \pm 0.60$ & $6.65-9.23$ & $8.15 \pm 0.41$ & $8.52-9.85$ & $9.11 \pm 0.51$ & $6.89-7.60$ & $7.33 \pm 0.43$ \\
\hline Harvest index & $35.24-49.00$ & $41.92 \pm 3.03$ & $\begin{array}{l}26.37- \\
34.85\end{array}$ & $30.59 \pm 2.97$ & $23.46-30.24$ & $27.72 \pm 2.09$ & $21.33-25.03$ & $23.46 \pm 1.11$ \\
\hline $\begin{array}{l}100 \text { seed } \\
\text { weight }\end{array}$ & $11.47-18.50$ & $14.78 \pm 1.07$ & $3.75-6.18$ & $5.13 \pm 0.52$ & $6.18-8.07$ & $7.15 \pm 0.36$ & $7.68-9.13$ & $8.96 \pm 0.22$ \\
\hline
\end{tabular}


Table.5 Simple correlation coefficients among different agro-morphological traits in soybean

\begin{tabular}{|c|c|c|c|c|c|c|c|c|c|c|c|c|c|c|}
\hline & $\begin{array}{r}\text { Days to } \\
50 \% \\
\text { flowering }\end{array}$ & $\begin{array}{r}\text { Days to } \\
75 \% \\
\text { maturity }\end{array}$ & $\begin{array}{r}\text { Reproductive } \\
\text { phase }\end{array}$ & $\begin{array}{r}\text { Plant } \\
\text { height }\end{array}$ & $\begin{array}{r}\text { Branches/ } \\
\text { plant }\end{array}$ & $\begin{array}{l}\text { Pods/ } \\
\text { plant }\end{array}$ & $\begin{array}{l}\text { Biological } \\
\text { yield/plant }\end{array}$ & $\begin{array}{r}\text { Harvest } \\
\text { index }\end{array}$ & $\begin{array}{r}\text { 100- } \\
\text { seed } \\
\text { weight }\end{array}$ & $\begin{array}{r}\text { Seeds/ } \\
\text { pod }\end{array}$ & $\begin{array}{r}\text { Nodes/main } \\
\text { stem }\end{array}$ & $\begin{array}{r}\text { Internode } \\
\text { length }\end{array}$ & $\begin{array}{r}\text { Petiole } \\
\text { length }\end{array}$ & $\begin{array}{r}\text { Pod } \\
\text { length }\end{array}$ \\
\hline $\begin{array}{l}\text { Seed } \\
\text { yield/plant }\end{array}$ & 0.1679 & 0.0596 & $0.7144 *$ & $0.6720^{*}$ & -0.3453 & $0.7841 *$ & 0.2809 & $0.8205^{*}$ & $0.6863^{*}$ & $0.0020^{-}$ & -0.2160 & $-0.5919 *$ & $0.7975^{*}$ & $0.5259 *$ \\
\hline $\begin{array}{l}\text { Days to } 50 \% \\
\text { flowering }\end{array}$ & & 0.4436 & 0.3926 & 0.1984 & -0.0678 & 0.0856 & $0.5678 *$ & -0.1421 & -0.0963 & 0.0298 & 0.0910 & 0.1597 & 0.0178 & 0.2729 \\
\hline $\begin{array}{l}\text { Days to } 75 \% \\
\text { maturity }\end{array}$ & & & 0.1278 & 0.2312 & 0.4578 & -0.2261 & 0.3014 & -0.0825 & -0.2583 & $0.1541^{-}$ & -0.1911 & 0.3139 & -0.2750 & -0.2319 \\
\hline $\begin{array}{l}\text { Reproductive } \\
\text { phase }\end{array}$ & & & & -0.4558 & -0.3837 & $0.5994^{*}$ & 0.4351 & 0.4864 & $0.6554 *$ & 0.1950 & -0.1338 & -0.4226 & $0.6672 *$ & $0.6820^{*}$ \\
\hline Plant height & & & & & 0.3355 & $0.8334^{*}$ & 0.2281 & $0.7893 *$ & $0.7089 *$ & 0.0536 & 0.2217 & $0.9232 *$ & $0.8698^{*}$ & -0.3188 \\
\hline Branches/plant & & & & & & -0.4936 & -0.1607 & -0.2439 & $0.5324^{-}$ & 0.3575 & 0.1188 & 0.3201 & $0.5660^{*}$ & $0.5731 *$ \\
\hline Pods/plant & & & & & & & 0.0837 & $0.7189 *$ & $0.7761^{*}$ & 0.0874 & -0.1028 & $-0.7889 *$ & $0.9494^{*}$ & $0.6072 *$ \\
\hline $\begin{array}{l}\text { Biological } \\
\text { yield/plant }\end{array}$ & & & & & & & & -0.3023 & 0.1584 & 0.1498 & 0.1471 & 0.1596 & 0.0704 & 0.4888 \\
\hline Harvest index & & & & & & & & & $0.5928^{*}$ & - & -0.3081 & $-0.6734 *$ & $0.7485^{*}$ & 0.2638 \\
\hline $\begin{array}{l}100 \text { seed } \\
\text { weight }\end{array}$ & & & & & & & & & & 0.1598 & -0.2188 & $-0.6430 *$ & $0.8880^{*}$ & $0.7135^{*}$ \\
\hline Seeds/pod & & & & & & & & & & & -0.0040 & -0.0933 & 0.1752 & 0.2750 \\
\hline $\begin{array}{l}\text { Nodes/main } \\
\text { stem }\end{array}$ & & & & & & & & & & & & -0.1521 & -0.1962 & -0.0542 \\
\hline $\begin{array}{l}\text { Internode } \\
\text { length }\end{array}$ & & & & & & & & & & & & & $0.8062^{-}$ & -0.3237 \\
\hline Petiole length & & & & & & & & & & & & & & $0.6823^{*}$ \\
\hline
\end{tabular}

* Significant at $\mathrm{P} \leq 0.05$ 
Grafius (1956) had also opined that the improvement of complex characters such as seed yield might be accomplished better through component breeding. Therefore, it is also important to gather information on association of yield with other characters and among themselves so as to form the basis to identify characters for increasing the efficiency of both direct and indirect selection and thereby defining an ideal plant type. Based on the estimates of correlation, the breeder will be able to decide the method of breeding to be followed to exploit the useful correlation.

In order to understand the nature and magnitude of correlations among seed yield per plant and other traits, estimates of simple correlation coefficients were computed for parents (cultivated and wild) and their interspecific crosses (Table 5).

Seed yield per plant exhibited significant and positive correlation with reproductive phase, pods per plant, harvest index, 100-seed weight, pod length and petiole length. Significant and positive correlation of seed yield with number of pods per plant and harvest index was observed by Barh et al., (2014) and for 100seed weight by Tomar et al., (2014). It was significantly negatively correlated with plant height and internode length.

Significant positive correlation was observed for days to $50 \%$ flowering and biological yield per plant. Reproductive phase has significant positive correlation with pods per plant, 100seed weight, petiole length and pod length. Plant height has positive correlation with internode length, whereas, negative correlation with pods per plant, harvest index, 100-seed weight and petiole length.

Branches per plant have negative correlation with 100-seed weight, petiole length and pod length. Pods per plant have positive correlation with harvest index, 100-seed weight, petiole length and pod length and negative correlation with internode length. Harvest index has positive correlation with 100 -seed weight and petiole length, whereas negative correlation with internode length. 100-seed weight has positive correlation with petiole length and pod length, whereas negative correlation with internode length.

Based on simple correlation studies, it can be concluded that seed yield per plant is positively correlated with reproductive phase, pods per plant, harvest index, 100-seed weight, pod length and petiole length and selection through these traits would be effective.

\section{Acknowledgment}

The first author is thankful to the Department of Science \& Technology, Ministry of Science \& Technology, Govt. of India for awarding the prestigious 'INSPIRE' fellowship during the study period to meet out the research expenses.

\section{References}

Barh A, Pushpendra, Singh K, Gupta MK, Joshi $\mathrm{M}$, et al., Genetic divergence and correlation studies on bhat (Black seeded soybean). Mitigating productivity constraints in soybean for sustainable agriculture. Proceedings of SOYCON 2014: International Soybean Research Conference. Society for Soybean Research and Development, Directorate of Soybean Research, Indore (India): p 111-112.

Carter T, Hymowitz T, Nelson R, et al., Biogeography, local adaptation, Vavilov, and genetic diversity in soybean. In Biological resources and migration. Berlin: Springer 2004. p. 47-59.

Chung G, Singh RJ. Broadening the Genetic Base of Soybean: A Multidisciplinary Approach. Crit Rev Plant Sci 2008; 27: 295-341.

Grafius JE. Components of yield in oats a geometrical interpretation. Agron J 1956; 48: 419-423.

Hammer KD. Domestications syndrome. Genet Resour Crop Evol 1984; 32: 11-34. 
Han Y, Zhao X, Liu D, Li Y, Lightfoot DA, Yang Z, Zhao L, Zhou G, Wang Z, Huang $\mathrm{L}$, et al., Domestication footprints anchor genomic regions of agronomic importance in soybeans. New Phytol 2016; 209: 871- 884.

Hartman GL, West ED, Herman TK, et al., Crops that feed the World 2. Soybeanworldwide production, use, and constraints caused by pathogens and pests. Food Security 2011; 3: 5-17.

Kabelka EA, Diers BW, Fehr WR, LeRoy AR, Baianu IC, You T, Neece DJ, Nelson RL, et al., Putative alleles for increased yield from soybean plant introductions. Crop Sci 2004; 44: 784-791.

Kim MY, Van K, Kang YJ, Kim KH, Lee SH, et al., Tracing soybean domestication history: from nucleotide to genome. Breed Sci 2012; 61: 445-452.

Lee J, Yu J, Hwang Y, Blake S, So Y, Lee G, Nguyen HT, Shannon JG, et al., Genetic diversity of wild soybean (Glycine soja Sieb. and Zucc.) accessions from South Korea and other countries. Crop Sci 2008; 48: 606-616.

Ma J, Nelson R. Evaluation of fertile lines derived from the hybridization of Glycine max and $G$. tomentella $(2 \mathrm{n}=78)$ a wild, perennial relative. M.Sc. thesis, 2012, University of Illinois at UrbanaChampaign, Madison, WI, USA.

Nakayama Y, Yamaguchi H. Natural hybridization in wild soybean (Glycine max subsp. soja) by pollen flow from cultivated soybean (Glycine max subsp. max) in a designed population. Weed Biol Manag 2002; 2: 25-30.

Singh RJ, Hymowitz T. The genomic relationship between Glycine max (L.) Merr. and Glycine soja Sieb. and Zucc. as revealed by pachytene chromosome analysis. Theor Appl Genet 1988; 76: 705-711.

Singh RJ, Kollipara KP, Hymowitz T, et al., Backcross-derived progeny from soybean and Glycine tomentella hayata intersubgeneric hybrids. Crop Sci 1990; 30: 871-874.

Song Q, Hyten DL, Jia G, Quigley CV, Fickus EW, Nelson RL, Cregan PB, et al., Fingerprinting soybean germplasm and its utility in genomic research. G3 (Bethesda) 2015; 5: 1999-2006.

Tomar K, Singh K, Pushpendra, Gupta MK, Yadav A, et al., Studies on genetic divergence in advanced breeding lines of soybean [Glycine max (L.) Merrill]. Mitigating productivity constraints in soybean for sustainable agriculture. Proceedings of SOYCON 2014: International Soybean Research Conference. Society for Soybean Research and Development, Directorate of Soybean Research, Indore (India): pp 109.

Weber CR. Inheritance and interrelation of some agronomic and chemical characters in an interspecific cross in soybeans, Glycine $\max x$ G. ussuriensis. Iowa Agric Expt Sta Res Bull 1950; 374: 765-816.

Zhou Z, Jiang Y, Wang Z, Gou Z, Lyu J, Li W, Yu Y, Shu L, Zhao Y, Ma Y, et al., Resequencing 302 wild and cultivated accessions identifies genes related to domestication and improvement in soybean. Nat Biotechnol 2015; 33: 408.

\section{How to cite this article:}

Indu Rialch and Jai Dev Sharma. 2019. Characterization of Interspecific Hybrids in Soybean (Glycine max L.). Int.J.Curr.Microbiol.App.Sci. 8(07): 2605-2617. doi: https://doi.org/10.20546/ijcmas.2019.807.321 